\title{
Suche nach der optimalen Bestrahlungsdosis bei NSCLC
}

\author{
Die gleichzeitige Radiochemotherapie ist Standard beim lokal fortgeschritte- \\ nen, nicht operablen nichtkleinzelligen Lungenkarzinom (NSCLC) im Stadium \\ III. Zum Nutzen einer höheren Bestrahlungsdosis liegen nun neue Ergebnisse \\ vor.
}

B isteir isher ging man davon aus, dass eine Steigerung der Bestrahlungsdosis bei der Radiochemotherapie des NSCLC die Chancen für das Überleben verbessert. Doch die Ergebnisse der großen PhaseIII-Studie RTOG 0617 zeigten das Gegenteil: Bei hochdosierter Bestrahlung sanken die Überlebensraten.

Um den Einfluss der Dosiseskalation auf das Gesamtüberleben (OS) bei Patienten mit NSCLC im Stadium III außerhalb kontrollierter randomisierter Studien $\mathrm{zu}$ untersuchen, wurde nun eine populationsbasierte Untersuchung anhand der Daten der nationalen Krebsda- tenbank der USA (National Cancer Database, NCDB) durchgeführt. 33.566 Patienten, die zwischen 2004 und 2012 eine Radiochemotherapie mit Bestrahlungsdosen zwischen 59,4 und 85 Gy erhalten hatten, wurden in die Studie eingeschlossen. Primärer Endpunkt war das OS.

Die Rate für das 2-Jahres-OS betrug in der Gesamtkohorte $43,1 \%$, für das 5-Jahres-OS 18,5\%. Die Analysen zeigten eine Verlängerung des OS mit steigender Dosis von median 18,8 Monaten bei 59,4-60 Gy auf 21,1 Monate bei 66 Gy, 22,0 Monate bei 70 Gy und 21,0 Mo- nate bei Dosen von $\geq 71$ Gy. Patienten, die mit 66,70 oder $\geq 71$ Gy bestrahlt wurden, hatten ein signifikant besseres OS als Patienten, die 59,4-60 Gy erhalten hatten $(\mathrm{p}<0,001)$. Dagegen bestand kein signifikanter Unterschied hinsichtlich des OS beim Vergleich von Bestrahlungen mit 66 Gy und $\geq 71$ Gy $(\mathrm{p}=0,38)$.

Fazit: Eine Steigerung der Bestrahlungsdosis über 60 Gy hinaus war mit einem verbesserten OS bei Patienten mit NSCLC im Stadium III assoziiert. Der Nutzen erreichte ein Plateau bei 66-70 Gy. Weitere Dosissteigerungen brachten keine weitere Verbesserung des OS.

Judith Neumaier

Brower JV et al. Improved survival with dose-escalated radiotherapy in stage III non-small-cell lung cancer: analysis of the National Cancer Database. Ann Oncol. 2016;27(10):1887-94.

\section{Afatinib bei NSCLC: Dosisreduktion beeinträchtigt Wirksamkeit nicht}

\begin{abstract}
Für Dosisreduktionen von Afatinib gibt es etablierte Protokolle. Doch wie wirken sie sich auf die Wirksamkeit und Verträglichkeit der Therapie bei Patienten mit nichtkleinzelligem Lungenkarzinom (NSCLC) aus?
\end{abstract}

A fatinib $40 \mathrm{mg} / \mathrm{d}$ ist beim NSCLC mit EGFR-Mutation für die Erstlinie zugelassen. Bei therapiebedingten Nebenwirkungen vom Grad $\geq 3$ oder bei bestimmten anhaltenden Nebenwirkungen vom Grad 2 kann die Dosis in 10-mgSchritten bis auf $20 \mathrm{mg}$ reduziert werden. Nun wurde der Einfluss der Dosisreduktion auf die unerwünschten Ereignisse, die Pharmakokinetik und das progressionsfreie Überleben (PFS) in den PhaseIII-Studien LUX-Lung 3 (weltweit) und 6 (Ostasien) untersucht. Alle therapienaiven Patienten mit fortgeschrittenem NSCLC mit EGFR-Mutation, die in diesen Studien Afatinib erhielten ( $\mathrm{n}=229$ bzw. 239), wurden in die Post-hoc-Analyse eingeschlossen. Die Dosis wurde bei 53,3\% der Patienten in LUX-Lung 3 und bei 28,0\% in LUX-Lung 6 reduziert, meist in den ersten 6 Monaten der Therapie. Bei Dosisreduktion nahm die Inzidenz therapiebedingter Nebenwirkungen von $100 \%$ auf 86,1\% (LUX-Lung 3) bzw. $74,6 \%$ (LUX-Lung 6) ab. Die Inzidenz

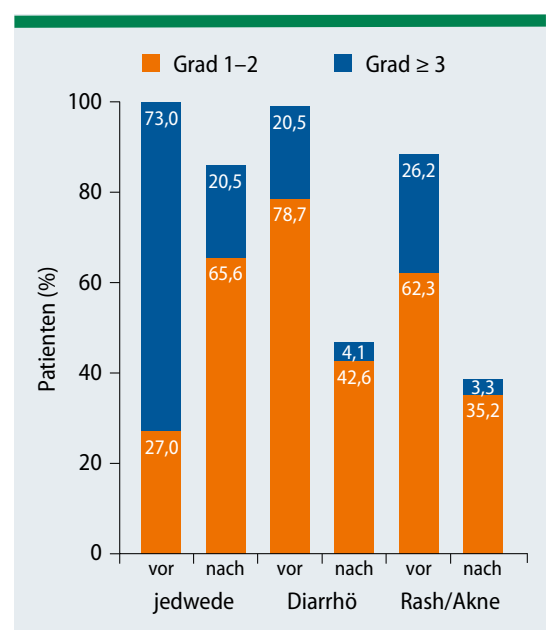

Abb. 1: Auswahl von häufigen Nebenwirkungen vor und nach der Dosisreduktion bei Patienten der LUX-Lung-3-Studie. von Nebenwirkungen vom Grad $\geq 3$ sank von $73 \%$ auf $20,5 \%$ (Abb. 1) bzw. von $80,6 \%$ auf $11,9 \%$. Patienten, die im Verlauf die Dosis reduzierten, hatten an Tag 22 höhere Afatinib-Plasmakonzentrationen als Patienten ohne Dosisreduktion (45,6 vs. 24,3 ng/ml). An Tag 43 waren die mittleren Konzentrationen in beiden Gruppen ähnlich (23,3 vs. $22,8 \mathrm{ng} / \mathrm{ml}$ ). Das mediane PFS war bei Patienten mit und ohne Dosisreduktion in den ersten 6 Monaten vergleichbar (LUX-Lung 3: 11,3 vs. 11,0 Monate; $\mathrm{p}=0,175$; LUX-Lung 6: 12,3 vs. 11,0 Monate; $p=0,982$ ).

Fazit: Beim NSCLC sind an die Verträglichkeit angepasste Dosisreduktionen eine effektive Maßnahme zur Reduktion therapiebedingter Nebenwirkungen unter Afatinib, die keinen Einfluss auf die therapeutische Wirksamkeit des Medikaments haben.

Judith Neumaier

Yang JCH et al. Effect of dose adjustment on the safety and efficacy of afatinib for EGFR mutation-positive lung adenocarcinoma: post hoc analyses of the randomized LUX-Lung 3 and 6 trials. Ann Oncol. 2016;27(11):2103-10. 